\title{
Potential Role of Hippo-Signaling Pathway in Gastric Cancer
}

\author{
Noman Ali', Muhammad Asim¹, Raheel Asghar1, Awais Amin², \\ Muhammad Saif Ur Rahman ${ }^{3 *}$ \\ ${ }^{1}$ Department of Bioinformatics \& Biotechnology, Government College University Faisalabad, \\ Allama Iqbal Road Faisalabad, Punjab, Pakistan \\ ${ }^{2}$ Department of Biomedical Engineering, College of Biomedical Engineering and Instrument Science, Zhejiang \\ University, Hangzhou, China \\ ${ }^{3}$ Clinical Research Center, The Second Affiliated Hospital, Zhejiang University School of Medicine, Hangzhou, \\ China \\ Email: *fiasanar@gmail.com
}

Received 28 February 2016; accepted 14 March 2016; published 18 March 2016

Copyright (C) 2016 by authors and OALib.

This work is licensed under the Creative Commons Attribution International License (CC BY).

http://creativecommons.org/licenses/by/4.0/

(c) () Open Access

\section{Abstract}

Gastric cancer is one of the most common malignancies worldwide. Understanding the molecular nature of gastric cancer is essential for the development of effective and personalized therapies. Several molecular signal transduction pathways drive tumorgenesis when deregulated and responded to different types of therapeutic interventions. The Hippo signaling pathway has been demonstrated to play a central role in the development and regulation of tissues and organ size. The deregulation of Hippo signaling leads to a concurrent combination of uncontrolled cellular proliferation and inhibition of apoptosis, two key hall marks in cancer development. The molecular nature of this pathway was first uncovered in Drosophila melanogaster through genetic screens to identify regulators of cell growth and cell division. The pathway is strongly conserved in humans, and rendering Drosophila is a suitable and efficient model system to better understand the molecular nature of this pathway. Current studies have demonstrated that a variety of deregulated molecules can alter Hippo signaling, leading to the constitutive activation of the transcriptional activator YAP or its paralog TAZ. Additionally, the Hippo-signaling pathway integrates inputs from a number of growth signaling pathways, positioning the Hippo-signaling pathway in a central role in the regulation of tissue size. Importantly, deregulated Hippo signaling is frequently observed in human cancers. YAP is commonly activated in a number of in vitro and in vivo models of tumorogenesis, as well as a number of gastric cancers. The common activation of YAP in many different tumor types and in gastric cancer provides an attractive target for potential therapeutic intervention. In this review, we appraise the evidence for the Hippo-signaling pathway as a cancer signaling network, and discuss cancer-relevant biological functions, potential mechanisms by which Hippo-signaling pathway activity is altered in cancer and emerging therapeutic strategies.

${ }^{*}$ Corresponding author. 


\title{
Keywords
}

\section{Gastric Cancer, Hippo Signaling, YAP (Yes-Associated Protein), Apoptosis}

\author{
Subject Areas: Oncology
}

\section{Introduction}

Gastric cancer is the fourth most regular perilous and second driving reason for death with more than 1.6 million new cases assessed in 2014 [1] [2]. The most well-known advancement of gastric tumor is gastritis metaplasiadysplasia carcinoma arrangement [3]. Early signs incorporate heart smolder, upper stomach agony, queasiness and loss of longing. Later signs and manifestations may incorporate weight reduction, yellow skin and whitening of the eyes, retching blood in the stool among others. The tumor might likewise spread from stomach to different parts of the body [4]. The most widely recognized reason is contamination by bacterium Helicobacter pylori, which reports in more than $60 \%$ of cases. Different sorts of $H$. pylori have more serious danger than others. Other basic reasons incorporate eating salted vegetables and smoking. Around $10 \%$ of cases are found in families and somewhere around $1 \%$ and $3 \%$ of cases are because of hereditary disorders acquired from a man's guardians as innate diffuse gastric growth. Most instances of stomach malignancies are gastric carcinomas. The larger part of gastric tumor is adenocarcinomas which are pathohistologically ordered into intestinal and diffuse sorts [5]. Gastric malignancy is additionally recognized by its huge geological varieties in rate and more than half of the aggregate gastric growth is in East Asian nations, for example, Japan, South Korea and China. Gastric cancer is a well-known reason for death in China with a predominance of 0.4 million instances of death every year [6] [7]. Gastric cancer progresses through a multistep process, the movement of which is variable impacted by both host hereditary and natural factors. Among those the gastric pathogen Helicobacter pylori is the most important component assuming adequately significant part in the improvement of both intestinal diffuse gastric non-cardia adenocarcinomas. There is preparatory confirmation that $H$. pylori diminishes the future danger [8]. In the event that tumor is dealt with ahead of schedule, numerous cases can be cured. Treatment may incorporate some kind of surgery, chemotherapy, radiations treatment and focus on treatment. On the off chance that late and calming consideration may be exhorted [9] [10]. As of late another way is found named as Hippo-signaling pathway flagging. Our capacity is to figure out these pathways that have both indicative and novel restorative focuses with which to treat the growth.

The Hippo-signaling pathway is the new found and saved signaling course, which manages organ size control by direct cell expansion and apoptosis. This study centered to investigate its consequences for human gastric disease. The Hippo-signaling pathway controls organ size in different species, though pathway deregulation can induce tumors in model living beings and happen in extensive variety of human carcinoma, including lung, liver colorectal and gastric malignancy [11] [12]. Regardless of this, physical or germ line changes in Hippo-signaling pathway qualities are exceptional, with just upstream pathway quality neurofibroin 2(NF2) perceived as real tumor silencer quality. Late studies in Drosophila melanogaster screens identified first four components of Hippo-signaling pathway, NDR family protein kinase warts (wts), the WW domain contains protein salvador, the ste-20 like protein kinase hippo (hpo) and adaptor protein Mob (Mps one binder) as tumor suppressor, which has an imperative part into counteract cell expansion and advancing apoptosis in separating epithelial cells [13] [14]. Much like malignancy cells, cells that contain changes for parts of the Hippo-signaling pathway multiply improperly and have a confrontational edge in hereditarily mosaic tissue. Albeit defectively indicated in warm blooded animals, a few parts of the Hippo-signaling pathway appear to be tumor silencers in people [15]. This review will cover the role of Hippo-signaling pathway in gastric cancer, its importance and future perspectives.

\section{Molecular Nature of Hippo Signaling:}

The fundamental sub-atomic building design of the hippo flagging pathway is rationed in the middle of Drosophila and humans. There is more prominent level of sub-atomic multifaceted nature and repetition seen in human Hippo-signaling pathway frequently with different mammalian homologs for a solitary Drosophila protein [16]. In humans, MST1/2(mammalian Ste20-like serine/threonine kinase), serve as upstream kinases that capacitate to 
phosphorylate Lats1/2. When the MST1/2 kinase course is inactivated, the YAP protein remains unphosphorylated, translocates into the core, and enacts interpretation of target qualities, and audited by Pobbati and Hong [17]. YAP does not have DNA typing capacities and requires a coupling accomplice to initiate quality interpretation. The most very much concentrated on transcriptional accomplices of YAP are the TEAD/TEF (Transcription enhancer factors) translation elements (TEAD 1 - 4) (Scalloped in Drosophila). The TEAD proteins can't actuate quality translation all alone, on the other hand, when YAP is available in the core they work together to advance interpretation of various proliferative and antiapoptotic qualities [18]. In spite of the fact that the relationship between YAP/TEAD is the best comprehended, YAP has likewise been shown to take up with extra DNA binding proteins amid transcriptional actuation, including SMAD family interpretation components. Not with standing the strong protection of central sub-atomic parts of the pathway, the transcriptional project of an enacted YAP/Yki, which advances cell multiplication and survival, is additionally saved [19]. In spite of the fact that flies and people accomplish this transcriptional program through the enactment of distinctive qualities, the cell result of the transcriptional profile stays moderate. The upstream controllers of the center Hpo/Wts kinase course incorporate the transmembrane protein fat and a few film related proteins. As an atypical cadherin, Fat (FAT1-4 in well evolved creatures) may work as a receptor, however an extracellular ligand has not been absolutely recognized. While Fat is known not to another atypical cadherin, Dachsous (Ds), amid tissue patterning [20], it is mystery what part Ds has in managing tissue development. By and by, Fat is perceived as an upstream controller of the Hpo pathway. Fat actuates Hpo through the apical protein Extended (Ex; FRMD6/Willin in well evolved creatures). Ex associates with two other apically-limited proteins, (KIBRA in warm blooded creatures) and Merlin (Mer; NF2 in vertebrates), to shape the Kibra-Ex-Mer (KEM) complex. Both Ex and Mer are FERM space containing proteins, while Kibra, as Sav, is a WW area containing protein. The KEM complex physically cooperates with the Hpo kinase course, in this manner limiting the center kinase cascade to the plasma layer for activation. Fat may likewise control Wts autonomously of Ex/Hpo, through the restraint of the unpredictable myosin Dachs. Ordinarily, Dachs can tie to and advance the debasement of Wts [21], as shown in Figure 1 [1].

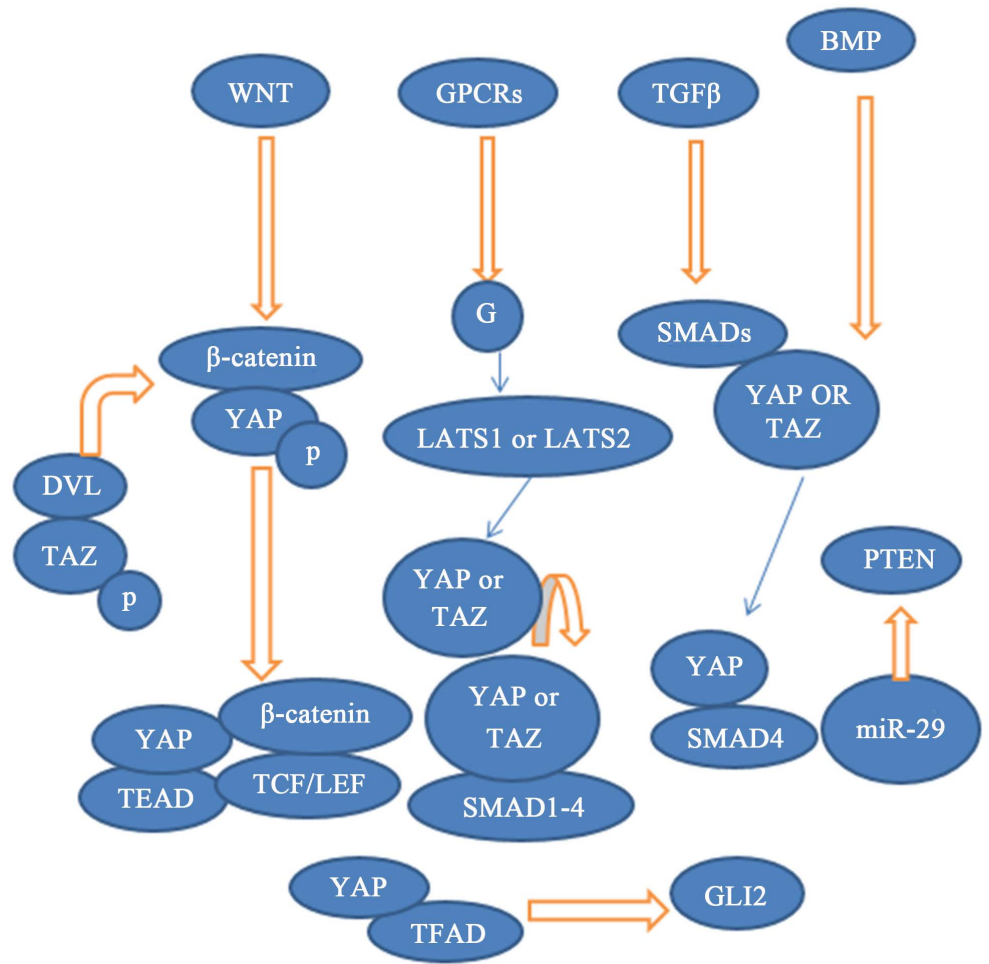

Figure 1. The figure shows that WNT activates Beta-cantenin associated with YAP which is phosphorylated which activates Beta-catenin, TCF, YAP and TEAD complex by attachment of DVL, TAZ(p) complex on the other hand GPCr Attached with G which activate LATS1 which further activates YAP and SMAD1 complex, YaP and TEAD complex finally activates GLI2.YAP and SMAD4 complex is activated by SMADs and YAP complex by TGFB and BPN while miR-29 activates PTEN. 


\section{Molecular Nature of Hippo-Signaling Pathway}

\subsection{Core Hippo-Signaling Pathway: A Kinase Cascade}

MST1/2 are STE20 (Serine/threonine) family protein kinases and can phosphorylate Sav1 (Single amino acid variant), Lats1/2(L-amino acid transporter) and Mob1. The kinase action of MST1/2 is upgraded through collaboration with Sav1, which is interceded by SARAH spaces present in both MST1/2 and Sav1.

MST1/2 straight forwardly phosphorylate Lats1/2 at the hydrophobic themes and this phosphorylation is required for Lats1/2 activation. Lats1/2 specifically connect with phosphorylated YAP/TAZ (Tafazzin) in which the collaboration may be interceded by PPxY theme on Lats1/2 and WW areas on YAP/TAZ.YAP/TAZ don't contain inherent DNA tying space however rather tie to the promters to target qualities by associating with DNA tying interpretation factors. YAP/TAZ basically ties to the translation components TEAD1-4 to direct gens included in cell expansion and cell demise [22].

\subsection{Cellular Inputs That Alter Hippo Signaling}

The center of the Hippo-signaling pathway, with a few kinases contrarily controlling YAP, is expanded by various diverse pathways that give info into this center cassete including cell extremity, cell to cell contact and G-protein coupled receptor flagging. Distinctive GPCR(G-protein coupled receptor) pathway have been indicated both initiative and restrain Yap contingent upon the particular G-protein enacted not withstanding modification on the center Hippo-signaling pathway, various other sub-atomic pathway generally changed in human carcinogenesis can cross converse with the Hippo-signaling pathway [23]. Wnt flagging is activated through cytoplasmic $\beta$-catenin. A transcriptional of $\beta$-catenin is CD44 which has the ability to initiate NF2, providing a system in which Wnt signaling can initiate the Hippo-signaling pathway. Wnt pathway changes have been appeared to expand the limitation of YAP, and YAP has been appeared to collaborate physically with $\beta$-catenin Hippo flagging is likewise directed in a post-translational way through the ubiquitination of Lats1 by the E3 ligase tingle [24]. Adjustments in the regulation of this post-translational change can deregulate the pathway. These studies point to a model, which includes a brought together part for the Hippo-signaling pathway in incorporating development signals from various pathways that manage tissue/organ size (Figure 2). Essentially, the across the board deregulation of a considerable lot of these pathways in human disease prompts the auxiliary inactivation of Hippo flagging, eventually adding to tumorogenesis (evaluated by Yu and Guan and investigated by Irvine) [25].

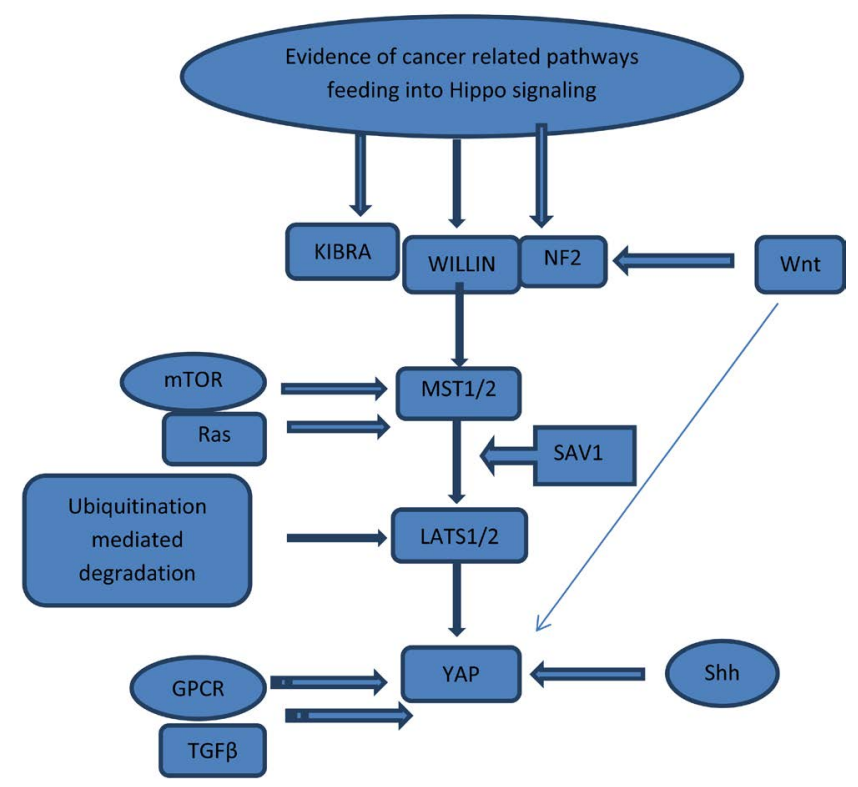

Figure 2. Flow sheet diagram of cancer related pathway that input into the Hipposignaling pathway. Showing core mammalian Hippo-signaling pathway components and additional cancer related molecular pathways. 


\subsection{The Hippo Signaling Pathway in Cancer}

In fruit fly, the Hippo flagging pathway includes a kinase course including the Salvador (Sav), Warts (Wts) and Hippo (Hpo) protein kinases. A significant number of the qualities included in the Hippo flagging pathway are perceived as tumor silencers, while Yki/YAP/TAZ is recognized as an oncogene [26]. This may be clarified by YAP's as of late characterized part in overcoming contact hindrance, a crucial development control property of ordinary cells in society in which expansion stops after cells reach confluence. This property is commonly lost in harmful cells, permitting them to multiply in an uncontrolled manner. Truth be told, YAP overexpression offends contact inhibition. Many of the pathway segments perceived as tumor silencer qualities are changed in human growths. For instance, changes in Fat4 have been found in bosom cancer, while NF2 is transformed in familial and sporadic schwannomas. Moreover, a few human disease cell lines summon transformations of the WW45 and MOBK1B proteins [27].

\subsection{Hippo-Signaling Pathway and Non Cancer Disease Process}

Not with standing its part in carcinogenesis, the Hippo-signaling pathway has been embroiled in Sjogren's disorder, an unending immune system issue bringing about the decimation of the salivary and lacrimal glands. In mice hippo flagging is required for typical salivary organs advancement and salivary organs from a mouse model sjogren's patients display atomic TAZ recoloring and upregulation of TAZ transcriptional targets. The Hipposignaling pathway is additionally embroiled in tissue recovery including a necessity for YAP in the recuperation of harmed mouse digestive tract YAP and TAZ have been appeared to be up-regulated in mouse wounds and thump down of YAP and TAZ deferrals wound conclusion [28] [29].

\section{Potential Therapeutics Targeting the Hippo-Signaling Pathway}

The Hippo-signaling pathway's commitment to malady pathogenesis has started enthusiasm for the advancement of potential therapeutics that could target key effectors of flagging cascade. No matter the component of Hippo-signaling pathway in initiation, growth cells much of the time display hyperactive YAP, suggesting that YAP is focal donor to tumorogenesis, Nonetheless YAP serves as alluring clinical focus to treat tumors with Hippo-signaling pathway deregulation. Of course early endeavors to create YAP, focusing on therapeutics have started [30]. Case in point, in a phone based screen, the medication dobutamine, a $\beta$-adrenergic receptor opponent, has been appeared to select YAP to the cytosol and hinder YAP-subordinate quality translation through a system inconsequential to center Hippo flagging. Also, a little particle medication named verteporfin, utilized as a part of the laser-enacted removal of veins in macular degeneration, has been recognized as an inhibitor of TEAD-YAP affiliation and YAP-affected liver abundance. As of late, verteporfin was appeared to stifle development in bosom malignancy cell lines, the cell lines with the most YAP expression were likewise the most delicate to verteporfin [31]. A recently portrayed tumor silencer quality named VGLL4 has been appeared to restrain the action of the YAP-TEAD complex by contending with YAP for tying to TEADs by means of pair tonduspaces, quite a peptide that emulates the YAP-TEAD inhibitor action of VGLL4 was appeared to stifle gastric tumor development in vitro and in vivo. Together, these early progressions in focusing on YAP have produced energy over imminent new therapeutics to treat malignancy and other ailment [32].

\section{Inhibitors Targeting Hippo-Signaling Pathway}

There are some compounds that currently involved in the inhibition of Hippo-signaling pathway some compounds are following that inhibits genes and proteins involved in Hippo-signaling pathway.

\begin{tabular}{ccc}
\hline Compounds & Target & Effect \\
\hline Verteporfin & YAP & Inhibit YAP-TEAD interaction and transcriptional activity in vitro \\
Dobutamine & B-adrenergic receptor agonist & $\begin{array}{c}\text { Causes the suppression and accumulation of } \\
\text { TEAD-YAP transcriptional activity in vitro }\end{array}$ \\
Botulinum toxin C3 & RHO & Inhibit YAP nuclear localization and TEAD activity \\
\hline
\end{tabular}




\section{Conclusion}

The Hippo-signaling pathway is a rationed flagging course that serves as a formative controller of organ size and when deregulated, it energizes carcinogenesis. Model frameworks, including Drosophila, mice, and cell society have given understanding into the atomic relationship of segments inside of the Hippo-signaling pathway and their control of cell division, apoptosis and at last, organ size. The Hippo-signaling pathway additionally coordinates signals from various built-up development pathways, including mTOR, Wnt, and Ras. Related to human malady, the deregulation of Hippo-signaling pathway segments advances disease improvement through various systems incorporating inactivating changes in upstream controllers, epigenetic modifications, loss of heterozygosity, adjustments in duplicate number, and deregulation of related atomic pathways. Despite their instrument, these adjustments yield the same atomic final result, the declaration of dynamic, atomic restricted YAP/TAZ. Focusing on the constitutive activation of YAP/TAZ for helpful treatment may address an assorted range of diverse sub-atomic changes piping into a typical transcriptional activator. This common atomic result presents both YAP and TAZ as appealing analytic and helpful markers for a variety of distinctive human malignancy.

\section{References}

[1] Statistics and Outlook for Stomach Cancer. Cancer Research UK. Retrieved on 19 February 2014.

[2] (2014) SEER Cancer Statistics FactSheets. http://seer.cancer.gov/statfacts/html/all.html

[3] Kang, J.M., Lee, B.H., Kim, N., et al. (2011) CDX1 and CDX2 Expression in Intestinal Metaplasia, Dysplasia and Gastric Cancer. Journal of Korean Medical Science, 26, 647-653. http://dx.doi.org/10.3346/jkms.2011.26.5.647

[4] Barros, R., da Costa, L.T., Pinto-de-Sousa, J., et al. (2011) CDX2 Auto-Regulation in Human Intestinal Metaplasia of the Stomach: Impact on the Stability of the Phenotype. Gut, 60, 290-298. http://dx.doi.org/10.1136/gut.2010.222323

[5] What Are the Risk Factors for Stomach Cancer (Website). American Cancer Society. Retrieved on 2010-03-31.

[6] Ruddon, R.W. (2007) Cancer Biology. 4th Edition, Oxford University Press, Oxford, 223.

[7] Orditura, M., Galizia, G., Sforza, V., Gambardella, V., Fabozzi, A., Laterza, M.M., Andreozzi, F., Ventriglia, J., Savastano, B., Mabilia, A., Lieto, E., Ciardiello, F. and De Vita, F. (2014) Treatment of Gastric Cancer. World Journal of Gastroenterology, 20, 1635-1649. http://dx.doi.org/10.3748/wjg.v20.i7.1635

[8] Saucedo, L.J. and Edgar, B.A. (2007) Filling out the Hippo-Signaling Pathway. Nature Reviews Molecular Cell Biology, 8, 613-621. http://dx.doi.org/10.1038/nrm2221

[9] Huang, M., Shen, A., Ding, J. and Geng, M. (2014) Molecularly Targeted Cancer Therapy: Some Lessons from the Past Decade. Trends in Pharmacological Sciences, 35, 41-50. http://dx.doi.org/10.1016/j.tips.2013.11.004

[10] Hariharan, I.K. (2006) Growth Regulation: A Beginning for the Hippo-Signaling Pathway. Current Biology, 16, R1037-R1039. http://dx.doi.org/10.1016/j.cub.2006.11.012

[11] Lai, Z.C., Wei, X., Shimizu, T., Ramos, E., Rohrbaugh, M., Nikolaidis, N., Ho, L.L. and Li, Y. (2005) Control of Cell Proliferation and Apoptosis by Mob as Tumor Suppressor, Mats. Cell, 120, 675-685. http://dx.doi.org/10.1016/j.cell.2004.12.036

[12] Basu, S., Totty, N.F., Irwin, M.S., Sudol, M. and Downward, J. (2003) Akt Phosphorylates the Yes-Associated Protein, YAP, to Induce Interaction with 14-3-3 and Attenuation of p73-Mediated Apoptosis. Molecular Cell, 11, 11-23. http://dx.doi.org/10.1016/S1097-2765(02)00776-1

[13] Vassilev, A., Kaneko, K.J., Shu, H., Zhao, Y. and De Pamphilis, M.L. (2001) TEAD/TEF Transcription Factors Utilize the Activation Domain of YAP65, a Src/Yes Associated Protein Localized in the Cytoplasm. Genes \& Development, 15, 1229-1241. http://dx.doi.org/10.1101/gad.888601

[14] Thompson, B.J. and Cohen, S.M. (2006) The Hippo-Signaling Pathway Regulates the Bantam microRNA to Control Cell Proliferation and Apoptosis in Drosophila. Cell, 126, 767-774. http://dx.doi.org/10.1016/j.cell.2006.07.013

[15] Yu, J., Zheng, Y., Dong, J., Klusza, S., Deng, W.M. and Pan, D. (2010) Kibra Functions as a Tumor Suppressor Protein That Regulates Hippo Signaling in Conjunction with Merlin and Expanded. Developmental Cell, 18, 288-299. http://dx.doi.org/10.1016/j.devcel.2009.12.012

[16] Yu, F.X. and Guan, K.L. (2013) The Hippo-Signaling Pathway: Regulators and Regulations. Genes \& Development, 27, 355-371. http://dx.doi.org/10.1101/gad.210773.112

[17] Yu, F.X., Zhao, B., Panupinthu, N., Jewell, J.L., Lian, I., Wang, L.H., Zhao, J., Yuan, H., Tumaneng, K., Li, H., Fu, X.D., Mills, G.B. and Guan, K.L. (2012) Regulation of the Hippo-YAP Pathway by G-Protein-Coupled Receptor Signaling. Cell, 150, 780-791. http://dx.doi.org/10.1016/j.cell.2012.06.037

[18] Irvine, K.D. (2012) Integration of Intercellular Signaling through the Hippo-Signaling Pathway. Seminars in Cell \& 
Developmental Biology, 23, 812-817. http://dx.doi.org/10.1016/j.semcdb.2012.04.006

[19] Schlegelmilch, K., Mohseni, M., Kirak, O., Pruszak, J., Rodriguez, J.R., Zhou, D., et al. (2011) Yap1 Acts Downstream of $\alpha$-Catenin to Control Epidermal Proliferation. Cell, 144, 782-795. http://dx.doi.org/10.1016/j.cell.2011.02.031

[20] Silvis, M.R., Kreger, B.T., Lien, W.-H., Klezovitch, O., Rudakova, G.M., Camargo, F.D., et al. (2011) $\alpha$-Catenin Is a Tumor Suppressor That Controls Cell Accumulation by Regulating the Localization and Activity of the Transcriptional Coactivator Yap1. Science Signaling, 4, ra33. http://dx.doi.org/10.1126/scisignal.2001823

[21] Cai, J., Zhang, N., Zheng, Y., de Wilde, R.F., Maitra, A. and Pan, D. (2010) The Hippo Signaling Pathway Restricts the Oncogenic Potential of an Intestinal Regeneration Program. Genes \& Development, 24, 2383-2388. http://dx.doi.org/10.1101/gad.1978810

[22] Enger, T.B., Samad-Zadeh, A., Bouchie, M.P., Skarstein, K., Galtung, H.K., Mera, T., Walker, J., Menko, A.S., Varelas, X., Faustman, D.L., Jensen, J.L. and Kukuruzinska, M.A. (2013) The Hippo Signaling Pathway Is Required for Salivary Gland Development and Its Dysregulation Is Associated with Sjogren’s Syndrome. Laboratory Investigation, 93, 1203-1218. http://dx.doi.org/10.1038/labinvest.2013.114

[23] Bertini, E., Oka, T., Sudol, M., Strano, S. and Blandino, G. (2009) YAP: At the Crossroad between Transformation and Tumor Suppression. Cell Cycle, 8, 49-57. http://dx.doi.org/10.4161/cc.8.1.7259

[24] Strano, S., Munarriz, E., Rossi, M., Castagnoli, L., Shaul, Y., Sacchi, A., Oren, M., Sudol, M., Cesareni, G. and Blandino, G. (2001) Physical Interaction with Yes-Associated Protein Enhances p73 Transcriptional Activity. The Journal of Biological Chemistry, 276, 15164-15173.

[25] Wang, H., Du, Y.C., Zhou, X.J., Liu, H. and Tang, S.C. (2014) The Dual Functions of YAP-1 to Promote and Inhibit Cell Growth in Human Malignancy. Cancer and Metastasis Reviews, 33, 173-181. http://dx.doi.org/10.1007/s10555-013-9463-3

[26] Hochhauser, J. and Tobias, D. (2010) Cancer and Its Management. 6th Edition, Wiley-Blackwell, Chichester, 259.

[27] Nomura, A., Grove, J.S., Stemmermann, G.N. and Severson, R.K. (1990) Cigarette Smoking and Stomach Cancer. Cancer Research, 50, 7084.

[28] Basu, S., Totty, N.F., Irwin, M.S., Sudol, M. and Downward, J. (2003) Akt Phosphorylates the Yes-Associated Protein, YAP, to Induce Interaction with 14-3-3 and Attenuation of p73-Mediated Apoptosis. Molecular Cell, 11, 11-23. http://dx.doi.org/10.1016/S1097-2765(02)00776-1

[29] Baumgartner, R., Poernbacher, I., Buser, N., Hafen, E. and Stocker, H. (2010) The WW Domain Protein Kibra Acts Upstream of Hippo in Drosophila. Developmental Cell, 18, 309-316. http://dx.doi.org/10.1016/j.devcel.2009.12.013

[30] Bazellieres, E., Assemat, E., Arsanto, J.P., Le Bivic, A. and Massey-Harroche, D. (2009) Crumbs Proteins in Epithelial Morphogenesis. Frontiers in Bioscience, 14, 2149-2169. http://dx.doi.org/10.2741/3368

[31] Wei, X., Shimizu, T. and Lai, Z.C. (2007) Mob as Tumor Suppressor Is Activated by Hippo Kinase for Growth Inhibition in Drosophila. The EMBO Journal, 26, 1772-1781. http://dx.doi.org/10.1038/sj.emboj.7601630

[32] Lee, M.J., Ran Byun, M., Furutani-Seiki, M., Hong, J.H. and Jung, H.S. (2014) YAP and TAZ Regulate Skin Wound Healing. Journal of Investigative Dermatology, 134, 518-525. http://dx.doi.org/10.1038/jid.2013.339 